\title{
Influência da atividade física nos sintomas depressivos de diabéticos e hipertensos
}

\section{Influence of physical activity on depressive symptoms of diabetic and hypertensive}

ARTIGO ORIGINAL | ORIGINAL ARTICLE

\begin{abstract}
RESUMO
A depressão é uma doença mental com uma elevada prevalência em doentes crónicos. Há evidências, na literatura, que a actividade física pode actuar como uma possível estratégia preventiva e terapêutica para os sintomas depressivos. O objectivo do nosso estudo foi investigar a relação existente entre actividade física e depressão num grupo de indivíduos com diabetes e hipertensão. Recolhemos uma amostra de 65 indivíduos, com idades compreendidas entre os 40 e os 94 anos, dos quais $41.5 \%$ são do sexo masculino. Os sintomas depressivos foram avaliados através do Patient Health Questionnaire-9. Não foi encontrada uma relação significativa entre actividade física e depressão. As mulheres apresentaram níveis depressivos mais elevados, cerca de duas vezes superiores aos homens. Verificámos, ainda, que quanto pior é a percepção de saúde maiores são os níveis de depressão.
\end{abstract}

Palavras-chave: actividade física, depressão, diabetes, hipertensão

\begin{abstract}
Depression is a mental illness with a high prevalence in chronic patients. There is evidence in the literature that physical activity can act as a possible preventative and treatment for depressive symptoms. Our study aimed to investigate the relationship between physical activity and depression in a group of individuals with diabetes and hypertension. The sample consisted of 65 individuals aged between 40 and 94 years, of which $41.5 \%$ are male. The Patient Health Questionnaire-9 was used to measure depressive symptoms. Results showed no statistically significant relationship between physical activity and depression. However, women presented higher levels of depression than men, about two times higher. It was also realized that worse levels of health perceptions also show higher levels of depression.
\end{abstract}

Keywords: physical activity, depression, diabetes, hypertension 
Segundo o DSM-V(American Psychiatric Association [APA], 2014), a Depressão Major caracteriza-se pela ocorrência dos seguintes sintomas: perda/ganho de peso, insónia/ hipersónia, agitação/lentificação psicomotora, fadiga/perda de energia, sentimento de inutilidade e de culpa em excesso, dificuldade de concentração, pensamento de morte/ideias suicidas; causando problemas a nível físico, psicológico e social.

Estima-se que as perturbações depressivas tenham uma elevada prevalência a nível mundial, surgindo em segundo lugar em termos de anos de vida perdidos devido a morte precoce ou incapacidade e sendo as mais prevalentes de entre as desordens psiquiátricas (Amaral et al., 2007; Helmich et al., 2010; Portal da Saúde, 2006a). Em Portugal, a depressão é responsável pela perda de mais de 1200 vidas por ano e um em cada cinco utentes dos cuidados de saúde primários portugueses encontra-se deprimido no momento da consulta (Portal da Saúde, 2006a). A Depressão é uma perturbação cujos sintomas estão muitas vezes mascarados (Ribeiro, 1998).

Helmich et al. (2010) referem que cerca de $25 \%$ das mulheres e $12 \%$ dos homens poderão vir a sofrer desta desordem ao longo do seu ciclo vital. As mulheres estão duas vezes mais predispostas a terem episódios depressivos do que os homens (APA, 2014; Portal da Saúde, 2006a). A incidência da depressão na população idosa varia entre 1 a $4 \%$ e duplica a partir dos 70 anos, não sendo maior do que na população em geral, mas pode chegar aos $14 \%$ em populações clínicas (Alexopoulos, 2005).

Há evidências que em populações clínicas a prevalência de perturbações depressivas é ainda mais elevada do que na população em geral (Goldney, Phillips, Fisher, \& Wilson, 2004), existindo uma "associação positiva entre depressão e o número de patologias crónicas" (Duarte \& Rego, 2007, p. 693). Dois exemplos destas patologias são a diabetes e a hipertensão arterial que surgem frequentemente associadas (Amaral et al., 2007).

Diabetes mellitus é uma doença crónica, caracterizada por hiperglicemia, isto é, a existência de níveis elevados de glucose no sangue (Arsa et al., 2009; MedlinePlus, s.d.; Farmácias Portuguesas, 2010). Cerca de 11\% a $15 \%$ dos pacientes com diabetes sofrem de Depressão Major. Esta encontra-se associada a uma menor aderência ao tratamento e a um menor envolvimento em atividades físicas (Acee, 2010; Steenbergen-Weijenburg et al., 2010; Wu, Parkerson, \& Doraiswamy, 2002).

A hipertensão arterial caracteriza-se pelas situações em que os valores de tensão arterial estão elevados, pode ser hipertensão arterial sistólica e hipertensão arterial diastólica. É uma doença crónica, mas que pode ser controlada. Na maioria dos casos, não são conhecidas as suas causas. Pode surgir em comorbidade como outra doença e estar associado à hereditariedade e à idade. Segundo Amaral et al. (2007), a prevalência da depressão em hipertensos é de 20\%. Em Portugal existem cerca de 2 milhões de hipertensos (Portal da Saúde, 2006b).

A depressão apresenta tanto sintomas somáticos como cognitivos. De entre os sintomas somáticos podemos relatar perturbações do sono, agitação ou lentificação psicomotora, fadiga, falta de energia, entre outros. Em relação aos sintomas cognitivos podemos referir o humor depressivo, sentimentos de inutilidade ou de culpa, dificuldades de concentração e pensamentos suicidas (Phelan et al., 2010; Smolderen et al., 2009; Stella, Gobbi, Corazza, \& Costa, 2002; Williams et al., 2009; Wu et al., 2002). Por vezes, os sintomas somáticos podem ser confundidos com os sintomas resultantes da doença e por esta razão não serem diagnosticados. De forma a ultrapassar estas reconhecidas dificuldades no diagnóstico, têm sido desenvolvidos e validados vários instrumentos psicométricos. De entre eles, tem merecido preferência 
pelos profissionais de saúde o "Patient Health Questionnaire”. Este revela fortes propriedades psicométricas para uso em populações clínicas, sendo um dos testes mais utilizados nestes contextos com o objetivo de uma maior precisão diagnóstica da depressão (Williams et al., 2009).

Outra variável relevante quando se fala em indivíduos com doenças crónicas é a percepção de saúde, que pode definir-se como uma avaliação subjetiva do estado de saúde (Jang, Kim, \& Chiriboga, 2006). De acordo com a literatura, uma percepção de saúde negativa encontra-se associada a maiores níveis de depressão (Jang et al., 2006; Maciel \& Guerra, 2006; Wu et al., 2002) e pessoas com mais do que uma doença crónica tendem a ter pior percepção de saúde (Jang et al., 2006).

As perturbações depressivas têm um impacto significativo na qualidade de vida das pessoas, acabando por agravar as condições inerentes à doença crónica (Garcia, Veiga, Motta, Moura, \& Casulari, 2010; Goldney et al., 2004). A doença crónica deixa o doente debilitado e com um "sentimento de incapacidade" (Godoy, Teixeira, Júnior, Michelli, \& Godoy, 2009, p.130), reforçando ou sendo reforçado pelos sintomas depressivos (Duarte \& Rego, 2007; Moraes et al., 2007).

Desta forma, é importante conhecer meios de melhorar a qualidade de vida destas pessoas que possuem uma comorbilidade entre a doença crónica (diabetes e hipertensão) e a Depressão.

Os tratamentos atualmente conhecidos por aliviar os sintomas, como por exemplo, a terapia farmacológica e a psicoterapia não apresentam resultados totalmente satisfatórios na remissão dos sintomas. São eficazes em alguns casos, mas não em todos. Para além disto, apresentam outras desvantagens, tais como o longo tratamento requerido em alguns casos, principalmente os mais graves, os efeitos adversos dos medicamentos e os custos elevados (Brosse, Sheets, Lett, \& Blumenthal, 2002).
Atualmente, há evidência científica que a atividade física é umas das melhores formas de manter a saúde física e mental (Helmich et al., 2010; Mello, Boscolo, Esteves, \& Tufik, 2005; Moraes et al., 2006). A atividade física é eficaz tanto na prevenção como no tratamento de perturbações depressivas. Investigações científicas demonstram que, para o tratamento de perturbações depressivas, uma terapia de atividade física regular é tão eficaz como a terapia farmacológica à base de antidepressivos (Babyak et al.,2000; Blumenthal et al.,2007; Helmich et al., 2010).

Quando a depressão surge associada a uma doença crónica, a prática de atividade física restabelece a autonomia e reduz o sofrimento do doente, contribuindo para a melhoria da sua qualidade de vida e, consequentemente, a redução dos sintomas depressivos (Godoy et al., 2009; Paulin, Brunetto, \& Carvalho, 2003).

Posto isto, o objetivo do nosso estudo foi verificar a prevalência de sintomas depressivos e analisar o efeito das variáveis sexo, idade e atividade física na depressão em indivíduos com uma doença crónica. Procurámos, ainda, relacionar a percepção de saúde com a depressão e, deste modo, averiguar se os indivíduos deprimidos têm uma pior percepção de saúde.

\section{MÉTODO}

O nosso estudo é de natureza quantitativa e transversal, uma vez que os nossos dados foram recolhidos num único momento. É ainda do tipo correlacional, porque temos como objectivo relacionar as variáveis, em estudo, entre si. O tipo de desenho utilizado é quasi-experimental, porque o grupo que constituí a amostra não foi escolhido aleatoriamente, já se encontrava formado no momento da recolha de dados (Sampieri, Collado, \& Lucio, 2006). A amostra foi recolhida com uma pré-condição: possuir uma doença crónica. 


\section{Amostra}

No presente estudo recolheu-se uma amostra clínica, onde os participantes tinham de ter necessariamente uma doença crónica. A amostra foi constituída por 65 indivíduos com diabetes e/ou hipertensão, sendo 41.5\% (27) do sexo masculino e $58.5 \%$ (38) do sexo feminino. As idades estão compreendidas entre os 40 e os 94 anos $(M=66.49, D P=10.42)$. De entre os indivíduos inquiridos $24.6 \%$ (16) são diabéticos, $53.8 \%$ (35) são hipertensos e $21.5 \%$ (14) sofrem de diabetes e hipertensão.

Como critério de exclusão foi considerado a incidência de outras patologias cuja manifestação sintomática ofereça algum tipo de confusão com as manifestações de depressão, como por exemplo a demência e/ou outras. Encontrámos também pessoas com traços/indícios de demência, as quais excluímos da amostra por fornecerem dados pouco fiáveis e claros.

\section{Identificação das variáveis}

De entre as variáveis independentes que constam no nosso estudo temos o sexo, a idade, tipo de doença, percepção de saúde e atividade física. Como variável dependente considerámos a depressão.

\section{Instrumentos}

Para esta investigação foi elaborado um questionário onde constam dados sócio-demográficos (sexo e idade); se toma antidepressivos, percepção de saúde (varia entre 1 e 10, sendo 1 "nada saudável" e 10 "muito saudável"), actividade física (número de dias em que praticou actividade física na última semana ou que pratica usualmente); e o Patient Health Questionnaire de 9 itens (PHQ-9), desenvolvido por Spitzer, Kroenke e Williams (1999, cit. por Williams et al., 2009) que foi traduzido e adaptado por nós, para o presente estudo. Deriva do Primary Care Evaluation of Mental Disorders (PRIME-MD) que foi desenvolvido para detectar: depressão, ansiedade, alcoolismo, transtorno somatoforme e perturbação alimentar (Zuithoff et al., 2010).
O PHQ-9 foi inicialmente validado para rastreio da Depressão e quantifica a frequência de 9 sintomas do diagnóstico do DSM-IV, nas 2 últimas semanas (Smolderen et al., 2009). É constituído por 9 itens, e cada um é avaliado numa escala de quatro pontos: 0- nenhum, 1alguns dias, 2- mais de metade dos dias, 3- quase todos os dias (Zuithoff et al., 2010).

A pontuação total é dada pela soma das pontuações de cada item, podendo variar entre 0 e 27. Uma pontuação entre 5 a 9 revela sintomas depressivos mínimos/ligeiros, entre 10 a 14 corresponde a sintomas moderados, entre 15 a 19 consideram-se sintomas depressivos moderadamente severos e uma pontuação superior ou igual a 20 representa uma depressão severa (Acee, 2010). Estes valores de critério foram propostos para amostras com nacionalidade diferente daquela que é objcto de análise no presente estudo. Assim, estes critérios requerem ser validados para a população portuguesa

Quatro dos itens estão associados a sintomas depressivos somáticos: perturbação do sono, cansaço, falta ou excesso de apetite e agitação ou lentificação psicomotora. Os outros 5 itens estão relacionados com falta de interesse, humor depressivo, sentimentos negativos sobre si mesmo, problemas de concentração e ideação suicida, e são classificados como sintomas depressivos cognitivos (Smolderen et al., 2009).

O PHQ-9 mostrou boa consistência interna e fiabilidade (Zuithof et al., 2010; Williams et al., 2009), e tem-se revelado um instrumento importante na identificação dos casos de risco elevado. Esta importância deve-se ao facto de ser um questionário de auto-relato de fácil aplicação e relativamente breve, sendo, por isso, facilmente utilizado numa entrevista de diagnóstico (Smolderen et al., 2009; Williams et al., 2009).

\section{Procedimentos}

Os participantes foram recrutados em duas instituições de saúde do Norte de Portugal. O grupo de trabalho obteve autorização através de um pedido escrito entregue ao diretor e à coorde- 
nadora de ambas as instituições que se disponibilizaram a colaborar no nosso estudo. Os questionários foram aplicados após a consulta dos pacientes. Dado que a amostra foi constituída maioritariamente por pessoas idosas analfabetas, as questões foram lidas e as respostas foram assinaladas pelos elementos do grupo.

Para a análise estatística dos dados utilizámos o Statistical Package For The Social Sciences (SPSS), versão 23. Inicialmente, efetuámos a análise ao nível da estatística descritiva para todas as variáveis. Para comparação das médias entre sexos foi utilizado o teste de Mann-Whitney para amostras independentes. Os participantes foram divididos em grupos de acordo com as idades. Indivíduos com mais de 65 anos (inclusive) foram considerados idosos e os restantes foram considerados adultos (Moraes et al., 2007). Para comparação das médias entre adultos e idosos recorremos ao teste de Mann-Whitney para amostras independentes. Para comparação das médias entre o grupo dos diabéticos, dos hipertensos e dos diabéticos e hipertensos recorremos ao teste Kruskal-Wallis.

De acordo com os níveis de atividade física, os indivíduos inicialmente foram divididos em duas categorias: os que atingem e os que não atingem as recomendações de atividade física. As recomendações de atividade física para pessoas diabéticas e hipertensas englobam no mínimo 30 minutos de atividade física (quer seja de forma contínua ou alternada) pelo menos 5 dias por semana, com o objectivo de manter ou melhorar a saúde (Gormley \& Hussey, 2005), à semelhança das recomendações de atividade física para a população em geral (American Health Association [AHA], 2017). As recomendações para adultos e idosos são semelhantes (Gormley \& Hussey, 2005). Para comparar os níveis depressivos entre os indivíduos que atingem e não atingem as recomendações foi aplicado um teste de Mann-Whitney para amostras independentes.

Posteriormente, a amostra foi dividida em 3 grupos: inativos (entenda-se por aqueles cuja actividade se limita às suas rotinas diárias), pouco ativos e muito ativos. Foram classificados como pouco ativos aqueles que praticavam no mínimo 30 minutos de atividade física (contínua ou alternada) entre 2 a 4 dias da semana. Foram considerados muito ativos aqueles que praticavam no mínimo 30 minutos de atividade física (contínua ou alternada) em pelo menos 5 dias por semana, o que coincide com as recomendações da AHA (2017). Para a comparação entre estes 3 grupos utilizámos a análise de Kruskal-Wallis.

Relativamente à percepção de saúde, agrupamos os indivíduos em 3 grupos: os que têm uma má percepção de saúde (entre 1-3), percepção de saúde intermédia (4-6) e boa percepção de saúde (7-10). Foi feita uma análise entre grupos utilizando o Kruskal-Wallis.

\section{Análise Estatística}

Inicialmente, usamos o teste de Levene skewness e curtose para verificar a homogeneidade das variâncias e ao skewness e cutose para a normalidade dos dados. Para a comparação entre grupos recorremos este de Mann-Whitney e Kruskall-Wallis.

\section{RESULTADOS}

O valor obtido para a nossa consistência interna, dado pelo alpha de Cronbach, foi de 0.83, o que nos leva a concluir que a nossa escala mantém uma boa consistência interna, tal como aconteceu com Ribeiro (2008). Após a análise do skewness e do kurtosis verificámos que a maioria dos valores não se situam entre -1 e 1 e, por esta razão, utilizámos testes não paramétricos (Dancey \& Reidy, 2006).

Os resultados obtidos evidenciaram que $72.3 \%$ (47) apresentam sintomas depressivos, sendo que apenas $7.7 \%$ (5) da amostra tem depressão severa. A maior percentagem de sintomas (41.5\%) situa-se nos sintomas depressivos ligeiros. Podemos referir ainda que $27.7 \%$ (18) indivíduos estão medicados com 
antidepressivos. Considerando os níveis recomendados de atividade física para este tipo de população $49.2 \%$ (32) indivíduos não atingem as recomendações e $50.8 \%$ (33) atingem as recomendações.

Através da análise das estatísticas descritivas podemos verificar que $81.6 \%$ das mulheres apresentam mais do dobro dos sintomas depressivos, que os homens $40.7 \%$.

De entre os 27 indivíduos do sexo masculino inquiridos, $44.4 \%$ (12) revelam sintomas depressivos ligeiros, dois apresentam sintomas depressivos moderados, um apresenta sintomas moderadamente severos e um sofre de depressão severa. No que diz respeito aos indivíduos do sexo feminino $39.5 \%$ (15) revelam sintomas depressivos ligeiros, $15.8 \%$ (6) apresentam sintomas depressivos moderados, $15.8 \%$ (6) apresentam sintomas moderadamente severos e $10.5 \%$ (4) têm depressão severa.

Os dados que constam no Quadro 1, obtidos através do teste de Mann-Whitney, mantendo um nível de significância de .05 , permitem-nos observar que existem diferenças estatisticamente significativas entre homens e mulheres no que diz respeito aos níveis de depressão, com as mulheres a apresentar níveis mais elevados, $\mathrm{U}_{(27,38)}=329.5, p=.01$.

Quadro 1

Níveis de depressão consoante o sexo (Mann-Whitney)

\begin{tabular}{lcc}
\multicolumn{2}{c}{ Sexo } & Níveis de depressão \\
\hline & M & $U$ \\
Masculino & 26.20 & $329.50^{*}$ \\
Feminino & 37.83 & \\
\hline${ }^{*} p<.05$ & &
\end{tabular}

De entre os 9 itens que constituem a nossa escala há diferenças significativas entre homens e mulheres quanto ao humor depressivo, $\mathrm{U}_{(27,38)}=315, p=.01$, distúrbios do sono, $\mathrm{U}_{(27,38)}=332, p=.01$, pouca energia, $\mathrm{U}_{(27,38)}=$
352.5, $p=.02$, distúrbios alimentares, $\mathrm{U}_{(27,38)}$ $=366, p=.03$ e agitação/lentificação psicomotora, $\mathrm{U}_{(27,38)}=371, p=.04$, em que as mulheres obtêm valores mais elevados, tal como é demonstrado no Quadro 2.

Quadro 2

Diferença entre sexos em alguns itens da escala

\begin{tabular}{lccc}
\hline & \multicolumn{2}{c}{ Sexo } & U \\
\cline { 2 - 3 } & Masculino & Feminino & M \\
\cline { 2 - 4 } & $\mathrm{M}$ & 38.21 & $315.00^{*}$ \\
Humor depressivo & 25.67 & 37.76 & $332.00^{*}$ \\
Distúrbios do sono & 26.30 & 37.22 & $352.50^{*}$ \\
Pouca energia & 27.06 & 36.87 & $366.00^{*}$ \\
Distúrbios alimentares & 27.56 & 36.74 & $371.00^{*}$ \\
Agitação/lentificação psicomotora & 27.74 & & \\
\hline
\end{tabular}
${ }^{*} p<.05$ 
Quanto ao tipo de doença, utilizando a Kruskal-Wallis $\chi^{2}$, mantendo um nível de significância de .05 , constatámos que não existem diferenças significativas entre indivíduos com diabetes, hipertensão ou ambas as doenças quanto aos níveis de depressão, $\chi^{2}$ (2) $=2.51$, $p=.29$. No entanto, verifica-se uma tendência para os indivíduos que sofrem de ambas as doenças apresentarem uma média superior em relação àqueles que sofrem apenas de um dos dois tipos de patologia referidos, como podemos observar no Quadro 3. Verificámos

Quadro 3

Tipo de doença e níveis de depressão (Kruskal-Wallis)

\begin{tabular}{lccc}
\hline & & \multicolumn{2}{c}{ Níveis de depressão } \\
\cline { 3 - 4 } Tipo de doença & N (65) & M & $\chi^{2}$ \\
\hline Diabetes & 16 & 29.56 & 2.51 \\
Hipertensão & 35 & 31.99 & \\
Diabetes e hipertensão & 14 & 39.46 & \\
\hline
\end{tabular}

ainda (quadro 4), utilizando a Kruskal-Wallis e mantendo um nível de significância de 5\%, que não há diferenças estatisticamente significativas entre diabéticos, hipertensos e indivíduos que possuem ambas as doenças no que diz respeito quer ao número de dias que praticam a atividade física, $\chi^{2}(2)=2.52, p=$
.28 , quer ao fato de atingirem ou não as recomendações de atividade física, $\chi^{2}{ }_{(2)}=3.47, p$ $=.18$. No entanto, verifica-se uma tendência para que indivíduos com ambas as doenças possuem uma média inferior no que concerne ao número de dias que praticam atividade física.

Quadro 4

Níveis de atividade física e níveis de depressão (Kruskal-Wallis)

\begin{tabular}{lccc}
\hline & & \multicolumn{2}{c}{ Níveis de depressão } \\
\cline { 2 - 3 } Níveis de atividade física & $\mathrm{N}(65)$ & $\mathrm{M}$ & $\chi^{2}$ \\
\hline Inativos & 22 & 35.14 & .70 \\
Pouco ativos & 10 & 29.50 & 32.64 \\
Muito ativos & 33 & 30 \\
\hline
\end{tabular}

Utilizando a Kruskal-Wallis $\chi^{2}$, com um nível de significância de $5 \%$ constatamos que não existem diferenças estatisticamente significativas entre adultos e idosos quanto à presença ou ausência de sintomas depressivos, $\chi^{2}(1)=$ $.07, p=.79$.

A análise dos dados apresentados no Quadro 5, utilizando a Kruskal-Wallis $\chi^{2}$, mantendo um nível de significância de .05 , indicou que não existem diferenças significativas, $\chi^{2}{ }_{(2)}=.7, p$ $=.7$, quanto aos níveis de depressão entre os grupos dos indivíduos considerados inativos, pouco ativos e muito ativos.

Comparando os níveis de depressão entre os indivíduos que atingem as recomendações de atividade física e aqueles que não atingem 
(quadro 4), utilizando o teste de Mann-Whitney, e mantendo um nível de significância de $5 \%$, verificamos que não existem diferenças significativas entre ambos os grupos, $\mathrm{U}_{(33,32)}=516, p=.87$.

Por fim, comparando a percepção de saúde com os níveis de depressão, utilizando a
Kruskal-Wallis, encontramos diferenças significativas entre os indivíduos com má, intermédia ou boa percepção de saúde, $\chi^{2}$ (2) $=$ 11.07, $p=.01$. Observa-se que quanto mais baixa é a percepção de saúde mais elevados tendem a ser os níveis de depressão.

Quadro 5

Percepção de saúde e níveis de depressão (Kruskal-Wallis)

\begin{tabular}{lccc}
\hline & & \multicolumn{2}{c}{ Níveis de depressão } \\
\cline { 3 - 4 } Percepção de saúde & N (65) & M & $\chi^{2}$ \\
\hline Má percepção & 8 & 43.38 & $11.07^{*}$ \\
Percepção intermédia & 38 & 36.32 & \\
Boa percepção & 19 & 22.00 & \\
${ }^{*} p<.05$ & &
\end{tabular}

\section{DISCUSSÃO}

O presente estudo visou comparar por sexo, idade, tipo de doença, percepção de saúde e prática de exercício físico sujeitos diagnosticados como diabetes e hipertensão. Os resultados encontrados na nossa amostra revelam uma grande prevalência de sintomas depressivos (72.3\%), o que representa um número elevado em relação ao que era esperado, tanto para diabéticos como para hipertensos.

Os idosos na sua maioria sofrem de várias patologias crónicas. Os clínicos não avaliam o indivíduo na sua totalidade, acabando por tratar as patologias individualmente, o que acarreta problemas no tratamento e acompanhamento, resultando daí comprometimento da saúde e da qualidade de vida (Duarte \& Rego, 2007). Por vezes, confundem os sintomas depressivos com doenças típicas da idade que apresentam sintomas semelhantes aos da depressão (Martins, s/d).

A comorbilidade entre patologias mentais, como a depressão, e doenças físicas como hipertensão e diabetes é frequente. A depressão pode levar a doenças crónicas e estas, por sua vez, podem piorar os sintomas depressivos (Duarte \& Rego, 2007). Segundo Amaral et al. (2007), um transtorno depressivo anterior pode ser um factor de risco para o aparecimento de hipertensão, se este tiver uma longa duração ou se se repetir por várias vezes. Também é de referir que o uso de determinados medicamentos como por exemplo anti-hipertensores, podem facilitar a ocorrência de episódios depressivos (Portal da Saúde, 2006b).

Embora a prevalência dos sintomas seja elevada tanto em homens $(40.7 \%)$ como em mulheres $(81.6 \%)$, estes resultados vão de encontro com a literatura, na medida em que a prevalência foi cerca de duas vezes superior nas mulheres (APA, 1994; Helmich et al., 2010; Portal da Saúde, 2006a).

Observa-se uma tendência para que os indivíduos que sofrem simultaneamente de diabetes e hipertensão tenham níveis depressivos mais elevados. Segundo Amaral et al. (2007) e Duarte e Rego (2007), esta tendência pode dever-se ao acumular de patologias que fragilizam o indivíduo tanto a nível físico como psicológico. Também verificámos uma menor tendência para a aderência à prática de atividade física entre os indivíduos com ambas as doenças crónicas. A doença crónica deixa o doente debilitado e com um sentimento de incapacidade. Quanto 
maior o acumular de patologias e as dificuldades sentidas maior será o sentimento de incapacidade, o que faz com o indivíduo vá desistindo de realizar as atividades quotidianas habituais (Godoy et al., 2009).

No nosso estudo, foi encontrada uma relação estatisticamente significativa entre percepção de saúde e depressão, em que indivíduos com percepção de saúde mais negativa tendem a ter níveis mais elevados de depressão, tal como foi observado por Jang et al. (2006), Maciel e Guerra (2006) e Wu et al. (2002).

As limitações causadas pelas doenças crónicas podem contribuir para denegrir a percepção de saúde, mas por outro lado, ter uma boa percepção de saúde pode contribuir para a aquisição e manutenção de uma maior saúde, quer física, quer mental (Jang et al., 2006). A percepção de saúde pode ser vista como um mediador entre a doença crónica e os sintomas depressivos. Simonsick, Wallace, Blazer, Berkman (1995, cit in Amaral et al., 2007) referem que os hipertensos podem apresentar sintomas depressivos por sentirem que a sua saúde está debilitada. Segundo Pais-Ribeiro, Silva, Meneses e Falco (2007) quanto mais optimista for a forma de encarar a doença, melhor será a percepção de saúde do indivíduo.

Ao contrário do que seria de esperar, não encontrámos uma relação significativa entre atividade física e depressão. A prática de atividade física não se encontrou relacionada com menores níveis depressivos, em nenhuma das circunstâncias analisada. Tanto os indivíduos que atingiam as recomendações como aqueles que não atingiam apresentavam níveis semelhantes de sintomas depressivos. O tipo de atividade física praticada pelos indivíduos da nossa amostra era essencialmente baseado em caminhadas, que estes faziam por prescrição médica, com o intuito de reduzir/melhorar os sintomas decorrentes das doenças crónicas. Este tipo de atividade física pode não se revelar totalmente eficaz na prevenção e no tratamento dos sintomas depressivos, por ser de baixa intensidade. Dunn, Trivedi, Kampert, Clarke e Chambliss (2005) efetuaram um estudo com 80 indivíduos e constataram que os participantes da condição de maior intensidade de exercício (17.5 kcal/kg/semana), de acordo com as recomendações de saúde pública, revelaram melhorias significativas nos sintomas depressivos, em comparação com o grupo que fazia parte da condição de baixa intensidade (7kcal/kg/semana).

Embora a média para os inativos fosse ligeiramente superior, esta não se revelou estatisticamente significativa. Este dado sugere que outras causas, em acréscimo à doença crónica, estejam por detrás do surgimento e manutenção dos sintomas, inibindo o efeito que possa ter a atividade física. Um exemplo é o luto (Stella et al., 2002). Constatámos, no decorrer do preenchimento dos questionários, que alguns indivíduos tinham perdido o marido ou a mulher há pouco tempo.

Também pode ter ocorrido uma confusão de sintomas. Por vezes, quando há patologias físicas associadas, e em populações mais idosas, os sintomas decorrentes da doença e resultantes do envelhecimento, confundem-se com os sintomas depressivos do tipo somático (Phelan et al., 2010; Williams et al., 2009; Wu et al., 2002).

Outro fator apontado por Moraes et al. (2007) é o fato de não se saber qual a relação causal entre atividade física e depressão. Há essencialmente duas perspetivas. Alguns autores consideram que não aderência à prática de atividade física pode contribuir tanto para o surgimento como para a manutenção dos sintomas. Outros afirmam que os sintomas depressivos, como a falta de energia e a anedonia, entre outros, podem interferir na motivação para a prática de atividade física.

Moraes et al. (2007) citam estudos que referem que o potencial terapêutico e preventivo da atividade física sobre a depressão ainda é controverso. Os nossos resultados são semelhantes aos obtidos nos estudos de Bailey e 
McLaren (2005) e Moraes et al. (2007) confirmando que não existem diferenças entre sujeitos ativos e inativos. Em parte, estas diferenças podem ser explicadas pela especificidade da amostra, nomeadaemnte ao nível de educação dos mesmos e por outro porque os sujeitos activos não seguiam qualquer plano de trabalho conceido por profissionais devidamente credenciados para o fazer. Mais, o nível de actividade física, no que se refere a volume e intensidade que nos foi descrito apresentava-se muito baixo $\mathrm{e}$, por isso, insuficientes para desencadear e impactar, adequadamente, os processos fisiológicos associados ao benefícios terapêuticos.

A nossa amostra foi constituída predominantemente por idosos, na maioria analfabetos, o que levou a que os questionários fossem preenchidos maioritariamente pelos autores deste estudo. À medida que colocávamos as questões, apercebíamo-nos que as pessoas revelavam dificuldades em fornecer respostas concretas, principalmente no que toca à questão da percepção de saúde, o que nos leva a colocar algumas reservas quanto à generalização dos resultados obtidos no que se refere à relação entre percepção de saúde e depressão, mesmo que os resultados por nós obtidos estejam de acordo com o descrito na literatura. Uma variável que poderá ser acrescentada em futuras investigações é o nível de escolarização. Colocamos a hipótese que a elevada prevalência de sintomas depressivos, entre outros fatores, possa ter alguma relação com o nível de escolarização, tal como foi mencionado noutros estudos (Jang et al., 2006; Maciel \& Guerra, 2006).

Percebemos também que a Depressão estava predominantemente associada a fatores como luto, solidão, dependência de terceiros, e acima de tudo por razões físicas (doenças crónicas e incapacitantes) e polipatologias. A Depressão em idosos tem características diferentes daquelas encontradas noutras faixas etárias.

Outra das limitações que apontamos para o nosso estudo, à semelhança de Moraes et al. (2007) é que a nossa medição dos níveis de ativi- dade física, pode ter resultado em erro, por se basear no auto-relato dos sujeitos, uma vez que os indivíduos podem ter conceitos diferentes do que é atividade física. Contudo, tentamos diminuir esta distorção dos resultados explicando aos sujeitos, no momento da aplicação do questionário, o que entendíamos por atividade física, dando exemplos.

No futuro, poderão ser efetuados estudos no sentido de inferir as relações causais entre percepção de saúde e depressão em indivíduos com doenças crónicas. Quanto à relação de atividade física com depressão, neste tipo de população, recomendamos que seja utilizada uma avaliação mais objetiva de atividade física.

\section{Agradecimentos: \\ Nada declarado.}

\section{Conflito de Interesses:}

Nada declarado.

\section{Financiamento:}

Nada declarado.

\section{REFERÊNCIAS}

Acee, A. (2010). Detecting and managing depression in type II diabetes: PHQ-9 is the answer! MEDSURG Nursing, 19(1), 32-38. PMID: 20336982

Alexopoulos, G. (2005). Depression in the elderly. Lancet, 365, 1961-1970.doi: 10.1016/S01406736(05)66665-2

Amaral, G. F., Jardim, P. C., Brasil, M. A., Souza, A. L., Freitas, H. F., ... , A. F., \& Ribeiro, C. N. (2007). Prevalência de transtorno depressivo maior em centro de referência no tratamento de hipertensão arterial. Revista de Psiquiatria do Rio Grande do Sul, 29(2), 161-168.

American Health Association (2017). Physical Activity and Public Health: Updated Recommendation for Adults From the American College of Sports Medicine and the American Heart Association. 
Circulation - Journal of the American Heart Association, 116, 1081-1093. doi: 10.1161/ CIR.0000000000000485

American Psychiatric Association (2014). Manual de Diagnóstico e Estatística das perturbações mentais (5 edição). Lisboa: Porto Editora

Arsa, G., Lima, L., Almeida, S. S., Moreira, S. R., Campbell, C. S., \& Simões, H. G. (2009). Diabetes Mellitus tipo 2: Aspectos fisiológicos, genéticos e formas de exercício físico para seu controle. Revista Brasileira Cineantropometria e Desempenho Humano, 11(1), 103-111.

Babyak, M., Blumenthal, J., Herman, S., Khatri, P., Doraiswamy, M., Moore, K., Craighead, E., Baldewicz, T. \& Krishnan, R. (2000). Exercise treatment for major depression: maintenance of therapeutic benefit at 10 months. Psychosomatic Medicine, 62, 633-638.

Bailey, M., \& McLaren, S. (2005). Physical activity alone and with others as predictors of sense of belonging and mental health in retirees. Aging E Mental Health, (1), 82-90.

Blumenthal, J., Babyak, M., Doraiswamy, M., Watkins, L., Hoffman, B., ... . \& Sherwood, A. (2007). Exercise and Pharmacotherapy in the Treatment of Major Depressive Disorder. Psychosomatic Medicine, 69, 587-596. doi: 10.1097/PSY.0b013e318148c19a

Brosse, A., Sheets, E., Lett, H. \& Blumenthal, J. (2002). Exercise and the treatment of clinical depression in adults. Sports Medicine, 32(12), 741-760. doi: 10.2165/00007256-20023212000001

Duarte, M., \& Rego, M. (2007). Comorbidade entre depressão e doenças clínicas em um ambulatório de geriatria. Cadernos de Saúde Pública, 23(3), 691-700. doi: 10.1590/S0102311X2007000300027.

Dunn, A.; Trivedi, M.; Kampert, J.; Clark, C. \& Chambliss, H. (2005). Exercise Treatment for Depression. American Journal of Preventive Medicine, 28(1), 1-8. doi: 10.1016/j. amepre.2004.09.003

Farmácias Portuguesas (2010, Outubro). Doce sabor amargo de boca. Farmácia Saúde, 170,
25-28.

Garcia, T., Veiga, J., Motta, L., Moura, F. \& Casulari, L. (2010). Depressed mood and poor quality of life in male patients with chronic renal failure undergoing hemodialysis. Revista Brasileira de Psiquiatria, 32 (4), 369-374. doi: 0.1590/S151644462010005000025.

Godoy, R., Teixeira, P., Júnior, B., Michelli, M., Godoy, D. (2009). Repercussões tardias de um programa de reabilitação pulmonar sobre os índices de ansiedade, depressão, qualidade de vida e desempenho físico em portadores de DPOC. Jornal Brasileiro de Pneumologia, 35(2), 129-136. doi: 10.1590/S180637132009000200005

Goldney, R., Phillips, P., Fisher, L. \& Wilson, D. (2004). Diabetes, depression and quality of life. Diabetes Care, 27, 1066-1070. doi: 10.2337/diacare.27.5.1066

Gormley, J. \& Hussey, J. (ed.) (2005). Exercise Therapy. Blackwell Publishing: Oxford.

Helmich, I., Latini, A., Sigwalt, A., Carta, M., Machado, S., Velasques, B., Ribeiro, P. \& Budde, H. (2010). Draft for Clinical Pratice and Epidemiology in Mental Health Neurobiological Alterations Induced by Exercise and Their Impat on Depressive Disorders. Clinical Pratice $\mathcal{E}$ Epidemiology in Mental Health, 6, 115-125. doi: 10.2174/1745017901107010106

Jang, Y., Kim, G. \& Chiriboga, D. (2006). Health perception and depressive symptoms among older Korean americans. Journal of CrossCultural Gerontology, 21, 91-102. doi: 10.1007/ s10823-006-9026-y

Maciel, A. \& Guerra, R. (2006). Prevalência e fatores associados à sintomatologia depressiva em idosos residentes no Nordeste do Brasil. Jornal Brasileiro de Psiquiatria, 55(1), 26-33. dsoi: 10.1590/S0047-20852006000100004.

Martins, R. (s/d). A depressão no idoso. Spectrum, 119-123.

MedlinePlus (s.d). Diabetes. Acedido a 8 de Março de 2011 em http://www.nlm.nih.gov/ medlineplus/diabetes.html 
Mello, M., Boscolo, R., Esteves, A., \& Tufik, S. (2005). O exercício físico e os aspetos psicobiológicos. Revista Brasileira de Medicina do Esporte, 11 (3), 203- 207. doi: 10.1590/S151786922005000300010.

Moraes, H., Deslandes, A., Ferreira, C., Pompeu, F. A., Ribeiro, P., \& Laks, J. (2007). O exercício físico no tratamento da depressão em idosos: revisão sistemática. Revista de Psiquiatria do Rio Grande do Sul, 29 (1), 70-79. doi; 10.1590/ S0101-81082007000100014.

Pais-Ribeiro, J., Silva, A., Meneses, R. \& Falco, C. (2007). Relationship between optimism, disease variables, and health perception and quality of life in individuals with epilepsy. Epilepsy \& Behavior, 11, 33-38. doi: 10.1016/j. yebeh.2007.04.010

Paulin, E., Brunetto, A., \& Carvalho, C. (2003). Efeitos de programa de exercícios físicos direccionado ao aumento da mobilidade torácica em pacientes portadores de doença pulmonar obstrutiva crónica. Jornal de Pneumologia, 29 (5), 287-294. doi: 10.1590/ S0102-35862003000500007

Phelan, E., Williams, B., Meeker1, K., Bonn, K., Frederick, J., LoGerfo, J., \& Snowden, M. (2010). A study of the diagnostic accuracy of the PHQ-9 in primary care elderly. Family Practice, 11(63), 1-9. doi: 10.1186/1471-229611-63

Portal da Saúde (2006a). Depressão. Obtido em http://www.min-saude.pt/portal/conteudos /enciclopedia $+\mathrm{da}+$ saude/saude + mental/ depressao.htm

Portal da Saúde (2006b). Hipertensão arterial. Obtido em http://www.portaldasaude.pt/ portal/conteudos/enciclopedia +da+saude/ doencas/doencas +do+aparelho+circulato rio/hipertensao+arterial.htm

Ribeiro, S. (1998). Atividade física e sua intervenção junto a depressão. Revista Brasileira Atividade Física \& Saúde, 3(2), 73-79.
Sampieri, R., Collado, C., \& Lucio, P. (2006, $3^{\text {a }}$ edição). Metodologia de pesquisa. McGraw-Hill Interamericana do Brasil Ltda: São Paulo.

Smolderen, K., Spertus, J., Reid, K., Buchanan, D., Krumholz, H., Denollet, J., Vaccarino, V., \& Chan, P. (2009, Abril, 25). The association of cognitive and somatic depressive symptoms with depression recognition and outcomes after myocardial infartion. Circulation Cardiovascular Quality and Outcomes, 328-337. doi: doi: 10.1161/ CIRCOUTCOMES.109.868588

Steenbergen-Weijenburg, K., Vroege, L., Ploeger, R., Brals, J., Vloedbeld, M., ... A., \& FeltzCornelis, C. (2010). Validation of the PHQ-9 as a screening instrument for depression in diabetes patients in specialized outpatient clinics. BMC Health Services Research, 10, 235-240. doi: 10.1186/1472-6963-10-235

Stella, F., Gobbi, S., Corazza D., \& Costa, J. (2002). Depressão no idoso: diagnóstico, tratamento e benefícios da atividade física. Revista Motriz, 8(3), 91-98.

Williams, R., Bode, R., Fann, J., Heinemann, A., Wilson, C., \& Tate, D. (2009). Improving Measurement Properties of the Patient Health Questionnaire-9 With Rating Scale Analysis. Rehabilitation Psychology, 54(2), 198-203. doi: 10.1037/a0015529

Wu, L., Parkerson, G., \& Doraiswamy, P. (2002). Health perception, pain, and disability as correlates of anxiety and depression symptoms in primary care patients. Journal of the American Board of Family Practice, 15, 183-190.

Zuithoff, N., Vergouwe, Y., King, M., Nazareth, I., Wezep, M., Moons, K., \& Geerlings, M. (2010). The Patient Health Questionnaire-9 for detection of major depressive disorder in primary care: consequences of current thresholds in a crosssectional study. BMC Family Practice. 